\title{
Correlation between Adiponectin and Triglyceride Levels in Pregnancy with and without Gestational Diabetes Mellitus
}

\author{
I Nyoman Gde Sudana ${ }^{1}$, Windarwati ${ }^{2}$, Budi Mulyono ${ }^{2}$ \\ ${ }^{1}$ Sanglah General Hospital Denpasar, Bali, Indonesia. E-mail: gdedana88@yahoo.com \\ ${ }^{2}$ Department of Clinical Pathology, Faculty of Medicine, Gadjah Mada University/Dr. Sardjito Hospital, Yogyakarta, Indonesia
}

\begin{abstract}
Gestational Diabetes Mellitus (GDM) is glucose intolerance during pregnancy. Adiponectin causes a decrease in glucose transporter 4 translocation, which reduces glucose uptake due to downstream insulin signal delivery and decreases non-esterified fatty acids so that triglyceride synthesis decreases in pregnancy. This study was conducted to determine the relationship between adiponectin and triglyceride levels in pregnancy, especially in a pregnant female with and without gestational diabetes mellitus. This observational cross-sectional study was conducted by measuring the levels of adiponectin and triglycerides in pregnancy, with and without gestational diabetes mellitus. Researchers proved by analyzing 75 subjects, 24-28 weeks pregnant females who were examined at the primary health center and Dr. Sardjito Hospital, Yogyakarta. Adiponectin levels have a moderate negative correlation with triglycerides in pregnant females $(r=-0.420$ and $p=0.002)$, a strong negative correlation with GDM ( $r=-0.680$ and $p=0.001)$, and moderate negative correlation ( $r=-0.455$, and $p=0.022$ ) with those without GDM. Based on this research can be concluded that adiponectin has a moderate negative correlation with triglycerides in pregnancy, a strong negative correlation in pregnant females with gestational diabetes mellitus, and a moderate negative correlation in those without gestational diabetes.
\end{abstract}

Keywords: Gestational diabetes mellitus, adiponectin, triglycerides

\section{INTRODUCTION}

Gestational Diabetes Mellitus (GDM) is a type of diabetes mellitus that is a carbohydrate tolerance disorder that results in increased blood glucose levels and was first recognized during pregnancy. The prevalence of GDM varies widely between 1-14\% of all pregnancies, and the frequency of undiagnosed GDM is around $10-25 \%$. The incidence of GDM is more common at the age of 32 years and more than $50 \%$ of patients have a family history of Diabetes Mellitus (DM), and about $40-60 \%$ of females who have experienced GDM after childbirth will develop type 2 diabetes or impaired glucose tolerance after 6 years of delivery. ${ }^{1}$ Pregnancy is a diabetogenic condition through the mechanism of insulin resistance with an increased compensatory response of cells and hyperinsulinemia. This insulin resistance plays a role to ensure an adequate supply of glucose for the fetus. ${ }^{2}$

Adiponectin is a physiologically active polypeptide hormone; its positive effect emerges by increasing the activity of Adenosine Monophosphate-Activated Protein Kinase (AMPK) in the liver and skeletal muscle and reducing glucose concentrations by inhibiting gluconeogenic enzymes and beta-oxidation of fatty acids. ${ }^{3}$ Adiponectin has an insulin-sensitizing effect by lowering tissue triglycerides. Tissue triglycerides play a role in insulin resistance due to their interference with the activation of insulin-stimulated phosphatidylinositol 3 kinase and Glucose Transporter 4 (GLUT-4) translocation and glucose uptake. This study aimed to determine the relationship between adiponectin and triglyceride levels in pregnant females with and without GDM.

\section{METHODS}

This study was an analytical observational study with a cross-sectional design to determine the correlation between adiponectin levels and triglyceride levels in pregnancy, a study of 24-28-week pregnant females with and without GDM. The research subjects were 24-28-week pregnant females who went to the public health center and Dr. Sardjito Hospital, Yogyakarta were selected consecutively and met the inclusion and exclusion criteria. The inclusion criteria in this study were 24-28-week pregnant females who were willing 
to take part in this study and agreed to the informed consent, while the exclusion criteria were patients who were previously diagnosed with diabetes, heart vascular disorders, a history of stroke and smoking. Patient data from history and physical examination included age, height, weight, number of pregnancies, previous history of DM, family history of diabetes, history of abortion, and history of giving birth to a baby $>4 \mathrm{~kg}$. This study has been approved by the Ethics Committee for Medical and Health Research, Faculty of Medicine, Public Health and Nursing, Gadjah Mada University, Yogyakarta with Ref number KE/FK/0949/EC/2020.

The results were first analyzed by using calibration tests, accuracy tests, and precision tests for method security. The characteristics of the research subjects were reported descriptively. The Kolmogorov-Smirnov test (sample size > 50) was used for the normality test for continuous data in this study. A p-value $>0.05$ indicated normal data distribution. Differences in the characteristics of the subjects were analyzed with one-way ANOVA and Kruskal-Wallis test for differences in three groups. The correlation between serum adiponectin levels and triglycerides was analyzed using the Pearson and Spearman correlation test. A significance limit of $p<0.05$ at a value with a $95 \%$ confidence interval was used for statistical analysis.

\section{RESULTS AND DISCUSSIONS}

The collection of research subjects lasted for three months, the subjects examined themself into several health centers in the Yogyakarta area including Umbulharjo Health Center, Kotagede Health Center, Mlati 1 Health Center, Wates Health Center, Pengasih Health Center, Pleret Health Center, Bambang Lipuro Health Center, and Dr. Sardjito Hospital. The characteristics of research subjects can be seen in Table 1.

The number of subjects who participated in the study was 100 pregnant females, but only 75 pregnant females who met the inclusion criteria were involved as the research subjects. In addition, 25 pregnant females were excluded from the study because 10 of them had a history of DM before pregnancy, 8 subjects had hypertension, 4 subjects had a history of smoking and 3 subjects had a history of stroke.

A total of 75 pregnant females who met the inclusion and exclusion criteria were involved as research subjects in this study as shown in Table 1. Pregnant females with older age were found in the GDM group compared to those without GDM. This result was different from the research conducted by Halil, which found no difference in age between the GDM group and the group of pregnant females

Table 1. Baseline characteristics of research subjects

\begin{tabular}{|c|c|c|}
\hline Variable & $\mathbf{n}$ & Value \\
\hline Age (years), mid (min-max) & 75 & $31(20-42)$ \\
\hline IMT $\left(\mathrm{kg} / \mathrm{m}^{2}\right)$ & & $26.59 \pm 4.69$ \\
\hline$<25 \mathrm{~kg} / \mathrm{m}^{2}(\%)$ & 29 & $41.3 \%$ \\
\hline$\geq 25 \mathrm{~kg} / \mathrm{m}^{2}(\%)$ & 46 & $58.7 \%$ \\
\hline \multicolumn{3}{|l|}{ Age of pregnancy } \\
\hline 24 weeks & 31 & $41.3 \%$ \\
\hline 25 weeks & 11 & $14.7 \%$ \\
\hline 26 weeks & 14 & $18.7 \%$ \\
\hline 27 weeks & 10 & $13.3 \%$ \\
\hline 28 weeks & 9 & $12 \%$ \\
\hline \multicolumn{3}{|l|}{ Laboratory test results } \\
\hline Fasting glucose (mg/dL), med (min-max) & & $72(53-123)$ \\
\hline 1-hour glucose (mg/dL), med (min-max) & & $125(64-227)$ \\
\hline 2-hour glucose $(\mathrm{mg} / \mathrm{dL})$, mean $\pm \mathrm{SD}$ & & $123 \pm 16$ \\
\hline Total cholesterol $(\mathrm{mg} / \mathrm{dL})$, mean $\pm \mathrm{SD}$ & & $227 \pm 48.3$ \\
\hline LDL cholesterol $(\mathrm{mg} / \mathrm{dL})$, mean $\pm \mathrm{SD}$ & & $137,9 \pm 52.1$ \\
\hline $\mathrm{HDL}$ cholesterol $(\mathrm{mg} / \mathrm{dL})$, mean $\pm \mathrm{SD}$ & & $57 \pm 11.8$ \\
\hline Triglyceride (mg/dL) med (min-max) & & $263(150-390)$ \\
\hline Adiponectin (ng/dL) med (min-max) & & $364,4(109,4-1031,8)$ \\
\hline
\end{tabular}

SD: Standard Deviation 
without GDM. ${ }^{4}$ The Body Mass Index (BMI) in a study by Leung was significantly higher in the group of pregnant females with GDM compared to that of without GDM, which was similar to this study. Contrastingly, a study by Altinova found no difference in BMI between the GDM and GDM groups. $^{5}$

Data subjects of this study with anthropometric characteristics shown in Table 2, where the medium age in the group of pregnant females with GDM is 33 (24-42) years, while pregnant females without GDM group was 29 (20-40) years. The characteristics of weight and BMI of the group of pregnant females with GDM in this study were 66 (42-91) kilograms and $28.3 \pm 4.88 \mathrm{~kg} / \mathrm{m}^{2}$ tended to be higher than the group of pregnant females without GDM, namely 59 $(33-78) \mathrm{kg}$ and $25.4 \pm 4.2 \mathrm{~kg} / \mathrm{m}^{2}$.

Based on WHO criteria on BMI, a body mass index of $25 \mathrm{~kg} / \mathrm{m}^{2}$ is classified as obesity/overweight and is a predisposing factor for the development of DM in general and GDM in particular. Patients with GDM have a higher BMI along with increased glucose indicating higher insulin resistance. Another study involving more than 1000 pregnant females showed that overweight/obese females at the time of pregnancy had increased risk factors for GDM along with increased BMI. The incidence of GDM is almost doubled in obese females compared to females whose a normal BMI. ${ }^{6}$

The biochemical characteristics of the markers of this study are shown in Table 3, where the group of pregnant females with DMG, medium fasting blood sugar levels were 81 (63-123) mg/dL, blood sugar levels 1-hour post-pandrial $180(94-227) \mathrm{mg} / \mathrm{dL}$, blood sugar levels 2-hours post-pandrial (2 hours PP) with a mean of $141 \pm 13 \mathrm{mg} / \mathrm{dL}$ while the group of pregnant females without medium DMG fasting blood sugar levels were 73 (54-88) $\mathrm{mg} / \mathrm{dL}$, blood sugar 1-hour post-pandrial 120 (64-160) mg/dL, blood sugar level 2-hours post-pandrial (2 hours PP) with a mean of $104 \pm 14 \mathrm{mg} / \mathrm{dL}$.

A study by Chatterjee reported that the average glucose levels 1 hour post-prandial in the group of pregnant females with GDM were $159 \pm 24.94 \mathrm{mg} / \mathrm{dL}$, while that of pregnant females without GDM were $100.3 \pm 15.17 \mathrm{mg} / \mathrm{dL}^{7}$. The average fasting glucose levels in this study were higher in the group of pregnant females with GDM than the group of pregnant females without GDM, suggesting similar results with other studies. The mean glucose values at 1-hour glucose and 2-hour glucose tests were significantly higher in pregnant females with GDM

Table 2. Comparison of anthropometric variables between pregnant females with and without GDM

\begin{tabular}{lllll}
\hline Variable & $\begin{array}{l}\text { Pregnant Females } \\
(\mathbf{n = 7 5 )}\end{array}$ & $\begin{array}{l}\text { With GDM } \\
(\mathbf{n = 2 5 )}\end{array}$ & $\begin{array}{l}\text { Without GDM } \\
(\mathbf{n = 5 0 )}\end{array}$ & p-value \\
\hline Age years med (min-max) & $31(20-42)$ & $33(24-42)$ & $29(20-40)$ & $0.01^{*}$ \\
BB kg med (min-max) & $63(33-91)$ & $66(42-91)$ & $59(33-78)$ & $0.015^{\star}$ \\
IMT kg/m mean \pm SD & $26.59 \pm 4.69$ & $28.3 \pm 4.88$ & $25.4 \pm 4.2$ & $0.024^{\star *}$ \\
Systole mmHg mean \pm SD & $114 \pm 15$ & $118,5 \pm 16.5$ & $110 \pm 14$ & 0.07 \\
Diastole mmHg mean \pm SD & $73 \pm 9$ & $75.5 \pm 9.8$ & $70 \pm 10$ & 0.068 \\
\hline
\end{tabular}

${ }^{*} \mathrm{p}<0.05$, Kruskal-Wallis test, ${ }^{* *} \mathrm{p}<0.05$, oneway ANOVA test, SD: Standard Deviation

Table 3. Comparison of laboratory parameters on 24-28-week pregnancy with and without GDM

\begin{tabular}{|c|c|c|c|c|}
\hline \multirow[b]{2}{*}{ Variable } & \multirow{2}{*}{$\begin{array}{c}\text { Pregnant } \\
\text { Females } \\
(n=75)\end{array}$} & \multicolumn{2}{|c|}{ Pregnant Females } & \multirow[b]{2}{*}{ p-value } \\
\hline & & $\begin{array}{c}\text { With DMG } \\
(n=25)\end{array}$ & $\begin{array}{l}\text { Without DMG } \\
\quad(n=50)\end{array}$ & \\
\hline $\mathrm{Hb} \mathrm{g} / \mathrm{dL}$ mean $\pm \mathrm{SD}$ & $11.6 \pm 0.8$ & $11.4 \pm 0.9$ & $12.45 \pm 0.78$ & 0.056 \\
\hline WBC $10^{3} / \mathrm{mmk}$ mean \pm SD & $9.98 \pm 2.3$ & $9.8 \pm 2.5$ & $10.14 \pm 2.15$ & 0.065 \\
\hline $\mathrm{PLT} 10^{3} / \mathrm{mmk}$ mean $\pm \mathrm{SD}$ & $278 \pm 71$ & $269 \pm 45$ & $292 \pm 60$ & $0.032^{\star *}$ \\
\hline GDP mg/dL med(min-max) & $72(53-123)$ & $81(63-123)$ & $73(54-88)$ & $0.01^{*}$ \\
\hline GD1PP mg/dL med (min-max) & $125(64-227)$ & $180(94-227)$ & $120(64-160)$ & $0.01^{*}$ \\
\hline GD2PP mg/dL mean $\pm S D$ & $123 \pm 16$ & $141 \pm 13$ & $104 \pm 14$ & $0.01^{\star *}$ \\
\hline Cholesterol $\mathrm{mg} / \mathrm{dL}$ mean $\pm \mathrm{SD}$ & $227 \pm 48.3$ & $214 \pm 10.7$ & $165 \pm 23$ & $0.026^{* *}$ \\
\hline $\mathrm{LDL} \mathrm{mg} / \mathrm{dL}$ mean $\pm \mathrm{SD}$ & $137,9 \pm 52.1$ & $149 \pm 13$ & $107 \pm 11$ & $0.025^{\star *}$ \\
\hline $\mathrm{HDL} \mathrm{mg} / \mathrm{dL}$ mean $\pm S D$ & $57 \pm 11.8$ & $55 \pm 10.6$ & $60 \pm 12$ & $0.04^{* *}$ \\
\hline $\mathrm{HbAlc} \%$ mean $\pm \mathrm{SD}$ & $5.5 \pm 0.4$ & $5.8 \pm 0.4$ & $5.32 \pm 0.3$ & 0.06 \\
\hline
\end{tabular}

${ }^{*} p<0.05$, Kruskal-Wallis test, ${ }^{* *} p<0.05$, oneway ANOVA test, SD: Standard Deviation 
Table 4. Difference of adiponectin and triglyceride levels in 24-28-week pregnancy with and without GDM

\begin{tabular}{lcllc}
\hline \multicolumn{1}{c}{ Variable } & Pregnant Females $(\mathbf{n}=\mathbf{7 5})$ & \multicolumn{1}{c}{ GDM $(\mathbf{n}=\mathbf{2 5})$} & \multicolumn{1}{c}{ Non-GDM $(\mathbf{n = 5 0})$} & \multicolumn{1}{c}{$\mathbf{p}$} \\
\hline Adiponectin $(\mathrm{ng} / \mathrm{mL})$ & $364,4(109,4-1.031,8)$ & $267,2(109,4-536,3)$ & $461,6(278-1.031,8)$ & $0.001^{*}$ \\
Triglyceride $(\mathrm{mg} / \mathrm{dL})$ & $263(150-390)$ & $288(200-319)$ & $238(150-390)$ & $0.009^{*}$ \\
\hline
\end{tabular}

${ }^{*} p<0.05$, Kruskal-Wallis test

than without GDM. Post-prandial hyperglycemia is associated with impaired insulin sensitivity of the mechanism of glucose transfer from mother to fetus. $^{7}$ A study by Bharathi reported that the total cholesterol lipid profile parameter values were significantly different between the groups of pregnant females, pregnant females with GDM and without GDM with total cholesterol levels of $256.5 \pm 41.7 \mathrm{mg} / \mathrm{dL}$, and $202.5 \pm 20.18 \mathrm{mg} / \mathrm{dL}$, respectively $(p<0.001)$. However, there was no difference between HDL and LDL levels between both groups. This study is also similar to a study by Fasshauer study, which reported higher LDL levels in the group of pregnant females with GDM and lower $\mathrm{HDL}$ levels in the group with GDM. ${ }^{8}$ During pregnancy, females with GDM have higher levels of LDL cholesterol and lower HDL cholesterol than healthy pregnant females. Human placental lactogen stimulates lipolysis, which results in high levels of circulating fatty acids including cholesterol. Increased stimulation of lipolysis results in disruption of cholesterol metabolism and glucose oxidation. An increased total and LDL cholesterol levels were found in GDM, associated with insulin resistance that occurs in GDM, which causes an increase in Free Fatty Acids (FFA), which are mobilized to the liver, resulting in the production of triglycerides and VLDL in the liver. Triglycerides in VLDL that enter the circulation will be exchanged with cholesteryl esters in the LDL core and hydrolyzed by lipoprotein lipase and liver lipase to form LDL. There is a lipid metabolism disorder in GDM, which is characterized by an increase in LDL and a decrease in HDL. ${ }^{9}$

Adiponectin levels in the group of pregnant females with GDM were significantly lower than the group of pregnant females and the group of pregnant females without GDM, suggesting a statistical difference between the three groups.

The unpaired comparative hypothesis of more than two groups of numerical variables was analyzed using the Kruskal-Wallis test because the data distribution was not normal. The results showed that the median of triglyceride levels in the group of pregnant females, group of pregnant females with GDM, and without GDM was 263 (150-390) $\mathrm{mg} / \mathrm{dL}$, 288 (200-419) mg/dL, and 238 (150-390) mg/dL, respectively with $p=0.009$, indicating a significant difference between the three groups (Table 4). The results of this study were in line with other studies. ${ }^{7.8}$ However, several studies have shown that there was no difference in adiponectin levels between GDM and non-GDM groups. ${ }^{10}$

Adiponectin has an effect on carbohydrate and fat metabolism, its concentration at 24-28 weeks of gestation is strongly affected by GDM associated with hormonal changes during pregnancy. This is due to insulin secretion induced by insulin resistance during pregnancy. ${ }^{8}$ Females with a history of GDM have been shown to have subclinical inflammation with elevated inflammatory markers such as TNF- $\alpha$. Tumor necrosis factor and other proinflammatory factors suppress the transcription of adiponectin, a key for insulin-sensitizing hormone. This hormone is produced by adipocytes. ${ }^{11}$

A study by Plows reported that adiponectin measurements had moderate predictive accuracy for the development of GDM, making it easier to determine target patients for screening with an Oral Glucose Tolerance Test (OGTT). This is relevant for countries where general screening for GDM is not carried out. Adiponectin examination in early pregnancy will provide an important diagnosis for pregnant women at high risk because adiponectin levels were found to be significantly lower than pregnant females without GDM. ${ }^{10}$

The results of this study were in line with a study by Bharathi study on 2017 reporting that triglyceride levels were significantly different between the group of pregnant females with GDM and without GDM with triglyceride levels in the group of pregnant females with DMG and the group of pregnant females were $286.4 \pm 77.6 \mathrm{mg} / \mathrm{dL}$ and $166 \pm 26 \mathrm{mg} / \mathrm{dL}$ with a significance value of $p<0.00113 .{ }^{6}$ Another study reported higher triglyceride levels in the group with GDM. Metabolic changes that occur in the liver and adipose tissue affect the levels of triglycerides, fatty acids, cholesterol, and phospholipids. After a decrease in the first 8 weeks of pregnancy, there will be an increase in triglycerides, fatty acids, cholesterol, lipoproteins, and phospholipids. Estrogen and insulin resistance cause hypertriglyceridemia in pregnancy. The mechanism of DMG induces dyslipidemia is in line with insulin resistance. During pregnancy, females with GDM 
Table 5. Comparison of correlation between adiponectin, triglyceride, and other cholesterol on 24-28-week pregnancy in GDM and non-GDM group

\begin{tabular}{lcccccc}
\hline & \multicolumn{7}{c}{ Adiponectin } \\
\hline & \multicolumn{7}{c}{ Pregnant Females } & \multicolumn{2}{c}{ Pregnant Females } \\
\cline { 2 - 7 } & $\mathrm{r}$ & $\mathrm{p}$ & $\mathrm{r}$ & $\mathrm{p}$ & $\mathrm{r}$ & $\mathrm{p}$ \\
\cline { 2 - 7 } & -0.420 & $0.002^{*}$ & -0.680 & $0.001^{*}$ & -0.455 & $0.022^{*}$ \\
Triglyceride & -0.169 & $0.024^{*}$ & -0.521 & $0.006^{*}$ & -0.511 & $0.009^{*}$ \\
Total cholesterol & -0.255 & $0.047^{*}$ & -0.533 & $0.006^{*}$ & -0.432 & $0.031^{*}$ \\
LDL & 0.384 & $0.006^{*}$ & 0.529 & $0.007^{*}$ & 0.447 & $0.025^{*}$ \\
HDL & & & & & & \\
\hline
\end{tabular}

${ }^{*} p<0.05$, Spearman test

have higher triglyceride levels than healthy pregnant people. ${ }^{12}$

Adiponectin has a negative correlation with triglycerides. In addition to influencing glucose metabolism and insulin sensitivity, adiponectin can modulate lipid levels in plasma. Other studies have also reported a negative correlation between adiponectin and triglycerides and LDL and a positive correlation with HDL. Adiponectin also regulates the metabolism of triglyceride-rich lipoproteins. Adiponectin increases the oxidation of fatty acids in circulation and skeletal muscle through the activation of AMP kinase. Therefore, low adiponectin levels will result in the accumulation of triglycerides. ${ }^{12}$

Adiponectin metabolism to triglycerides involves intrinsic changes in fat metabolism in skeletal muscle and affects lipoprotein lipase activity in skeletal muscle and adipocytes. Adiponectin can reduce triglyceride accumulation in skeletal muscle by increasing fatty acid oxidation through activation of acetyl CoA oxidase and AMP kinase. Adiponectin also stimulates lipoprotein lipase, a lipolytic enzyme that can catabolize VLDL through increased expression of peroxisome proliferators activator receptors in the liver and adipocytes. At the hepatic level, adiponectin can reduce the supply of non-esterified fatty acid in the liver in the gluconeogenesis process, resulting in a decrease in triglyceride synthesis. Low adiponectin levels and dyslipidemia in GDM patients are associated with lipoprotein lipase and pregnancy hormone levels. The effect of adiponectin affects the regulation of hepatic lipase activity in DMG patients, which explains the action of adiponectin in increasing $\mathrm{HDL}$ levels. ${ }^{13}$

This study found a negative correlation between adiponectin and triglycerides, total cholesterol, and LDL levels and a positive correlation between adiponectin and $\mathrm{HDL}$ in the group of pregnant females, pregnant females with and without GDM.
The results of this study were consistent with other studies that reported a correlation between adiponectin and triglycerides in pregnant females with GDM. Many of these studies found a negative correlation between adiponectin and triglycerides. This is supported by Retnakaran who reported that adiponectin is affected by lipolysis in GDM but is not affected by gluconeogenesis. ${ }^{14}$

The strength of the correlation between adiponectin and triglycerides between the groups from the strongest and significant negative correlation was found in the group of pregnant females with GDM ( $r=-0.680, p=0.001)$, moderate and significant strength in the group without GDM $(r=-0.455, p=0.022)$ and in the group of pregnant females $(r=-0.420, p=0.002) \quad($ Table 5). The correlation between adiponectin and triglycerides in the group of pregnant women with GDM was strongly negative, indicating that a lower adiponectin level led to a higher triglyceride level, which can be seen in Figure 1.

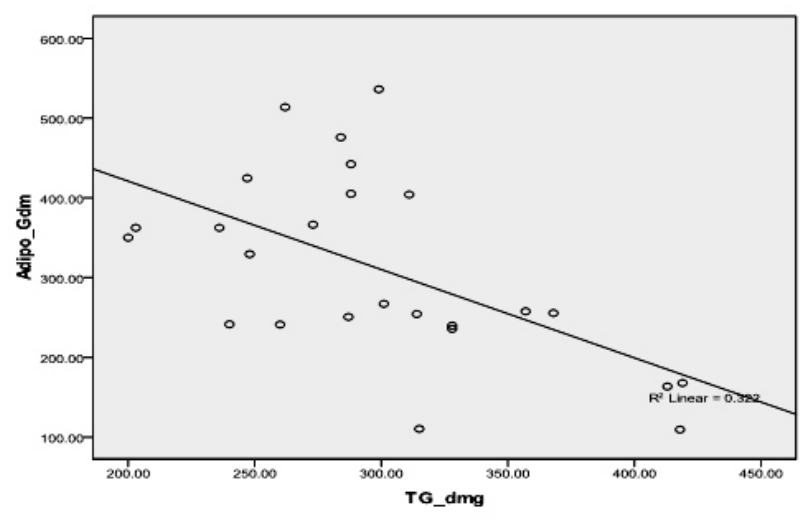

Figure 1. Correlation curve between adiponectin and triglyceride levels among pregnant females with GDM

The results of the correlation test in this study with linear regression R2 0.322 showed that the decrease in adiponectin levels was only $32.2 \%$ 
influenced by an increase in triglycerides. Other factors can affect adiponectin concentration including glucose levels. The glucose threshold for the establishment of GDM also varies with each criteria. In addition, adiponectin levels are also influenced by various things, including smoking, diet, BMI, maternal age, physical activity, insulin, and TNF. Smoking can affect adiponectin levels by provoking oxidative stress and inflammatory cytokines that can decrease adiponectin expression and secretion. Nicotine induces lipolysis and can suppress adiponectin gene expression., ${ }^{3,5}$ Pregnant females with smoking habits were excluded in this study; therefore, changes in adiponectin levels caused by smoking were able to be avoided.

A diet with foods that have a high glycemic index lowers adiponectin levels. The glycemic index affects adiponectin levels by increasing blood glucose. Blood glucose levels are inversely related to adiponectin expression in adipose tissue. ${ }^{6.7}$ However, this study did not assess the diet of pregnant females. Pregnant females were asked to fast 8-12 hours before sampling. The diet of pregnant females may affect adiponectin values.

Adiponectin levels are inversely related to obesity and decrease in people with increased adiposity through downregulated receptors. Physical activity can increase adiponectin levels. ${ }^{9}$ Adiponectin levels increase with physical activity and increase adiponectin receptor mRNA expression in skeletal muscle. ${ }^{13}$ Adiponectin is positively correlated with age. Older people have higher adiponectin levels. The high adiponectin secretion is in response to the detrimental processes caused by aging. ${ }^{14}$

The condition of adiponectin resistance can be explained in a study involving insulin-resistant mice with hyperadiponectinemia but with normal levels of adiponectin receptors 1 and 2, making them unable to lower blood glucose levels. ${ }^{4,5}$ Insulin and TNF measurements were not carried out in this study, making it possible for both parameters to affect the adiponectin results. As one of the hormones that influence the development of GDM, there are inconsistencies in determining the relationship between low adiponectin levels and an increased risk of GDM.

The limitations of this study were the absence of adiponectin and triglyceride assessments in the group of pregnant females with homogeneous subject age, assessment of diet by study subjects, assessment of body fat, physical activity, and measurement of insulin and TNF- $\alpha$.

\section{CONCLUSIONS AND SUGGESTIONS}

Based on the results of this study, it was concluded that there was a significant negative correlation between adiponectin and triglyceride levels in pregnant females, there was a significant negative correlation between adiponectin and triglyceride levels in pregnant females with GDM and there was a significant negative correlation between adiponectin and triglyceride levels in pregnant females without GDM.

Further research was needed on the relationship between adiponectin levels and other factors in the development of GDM with homogeneous age of pregnant females, similar diet and physical activity control, as well as insulin and TNF- $\alpha$ measurements.

\section{REFERENCES}

1. Rahayu A, Rodiani. Indonesian clinical practice guidelines for diabetes in pregnancy. JAFES, 2016; 28: 1-6.

2. Rahmawati F, Natosba J, Jaji. Intermediate metabolism in normal pregnancy and in gestational diabetes. Diabetes Metab Res Rev, 2016; 19: 259-270.

3. Thagaard IN, Krebs L, Holm JC, Lange T, Larsen T, Christiansen M. Adiponectin and leptin as first trimester markers for gestational diabetes mellitus: A cohort study. Clin Chem Lab Med, 2017; 0427: 1-6.

4. Pala HG, Ozalp Y, Yenner AS, Gercekliolu G, Uysal S, Onvural A. Adiponectin levels in gestational diabetes mellitus and in pregnant women without glucose intolerance. Adv Clin Exp Med, 2015; 24, 85-92.

5. Leung K, Draper JT, Boeras C, Min SY, Rojas-Rodriguez R, et al. Human adipose tissue expansion in pregnancy is impaired in gestational diabetes mellitus. Diabetologia, 2015; 58(9): 2106-14.

6. Bharathi KR, Vijayalakshmi S, Shrunga RP. Original research article a study of lipid parameters among GDM and non-GDM pregnant women: A hospital based study. Int J Reprod Contracept Obstet Gynecol, 2017; 6(12): 5488-90.

7. Chatterjee VKK, Wareham NJ, Rahilly SO. Elevated plasma adiponectin in humans with genetically defective insulin receptors. J Clin Endocrinol Metab, 2017; 91: 3219-23.

8. Brand-Miller JC, Thomas M, Swan V, Ahmad ZI, Petocz P, Colagiuri, S. Physiological Validation of the Concept of Glycemic Load in Lean Young Adults. Int J Nutr, 2018; 133(9): 2728-2732.

9. Bhavadharini $B$, Uma $R$, Saravanan $P$, Mohan V. Screening and diagnosis of gestational diabetes mellitus-relevance to low and middle income countries. J Clin Endocrinol Diabetes, 2016; 2(1): 13.

10. Plows JF, Stanley JL, Baker PN, Reynolds CM, Vickers $\mathrm{MH}$. The pathophysiology of gestational diabetes mellitus. Int J Mol Sci, 2018; 19: 1-21. 
11. Hernandez TL, Pelt RE. Van, Anderson MA, Reece MS, Reynolds RM, et al. Women with gestational diabetes mellitus randomized to a higher-complex carbohydrate/low-fat diet manifest lower adipose tissue insulin resistance, inflammation, glucose, and free fatty acids: A pilot study. Diabetes Care, 2016; 39: 39-42.

12. Ott R, Stupin JH, Melchlor K, Schellong K, Thomas Ziska $\mathrm{T}$, et al. Alteration of adiponectin gene expression and dna methylation in adipose tissues and blood cells are associated with gestational diabetes and neonatal outcome. Clinical Epigenets, 2018; 10: 131-142.

13. Stewart A, Malhotra A. Gestational diabetes and the neonate: Challenges and solutions. Res Rep Neonatol, 2015; 31-39.

14. Ashcroft FM, Rohm M, Clark A, Brereton MF. Is type 2 diabetes a glycogen storage disease of pancreatic $\beta$-cells?. Cell Metab, 2017; 26(1): 17-23. 